\title{
La Nativité italienne : une histoire d'adoration (1250-1450).
}

Thèse de doctorat d'italien, sous la direction d'Anna Fontes-Barato, université Sorbonne Nouvelle - Paris 3. Soutenue le 24 novembre 2012

\section{Giulia Puma}

\section{(2) OpenEdition} Journals

Édition électronique

URL : https://journals.openedition.org/cem/13207

DOI : $10.4000 /$ cem. 13207

ISSN : 1954-3093

Éditeur

Centre d'études médiévales Saint-Germain d'Auxerre

Référence électronique

Giulia Puma, "La Nativité italienne : une histoire d'adoration (1250-1450). », Bulletin du centre d'études médiévales d'Auxerre | BUCEMA [En ligne], 17.2 | 2013, mis en ligne le 02 décembre 2013, consulté le 22 septembre 2022. URL : http://journals.openedition.org/cem/13207 ; DOI : https://doi.org/10.4000/ cem. 13207

Ce document a été généré automatiquement le 22 septembre 2022.

\section{c) (i) (2)(2)}

Creative Commons - Attribution - Pas d'Utilisation Commerciale - Partage dans les Mêmes Conditions 4.0 International - CC BY-NC-SA 4.0

https://creativecommons.org/licenses/by-nc-sa/4.0/ 


\section{La Nativité italienne : une histoire d'adoration (1250-1450).}

Thèse de doctorat d'italien, sous la direction d'Anna Fontes-Barato, université Sorbonne Nouvelle - Paris 3. Soutenue le 24 novembre 2012

\section{Giulia Puma}

1 Cette thèse porte sur l'iconographie de la Nativité du Christ dans la peinture italienne médiévale, avec un ensemble de trois cents images incluant les petits retables destinés à la dévotion privée comme les grands cycles de fresques. L'enquête commence autour de 1250 par les productions des peintres qui précédèrent Giotto et Duccio et s'achève autour de 1450, avec les œuvres de Beato

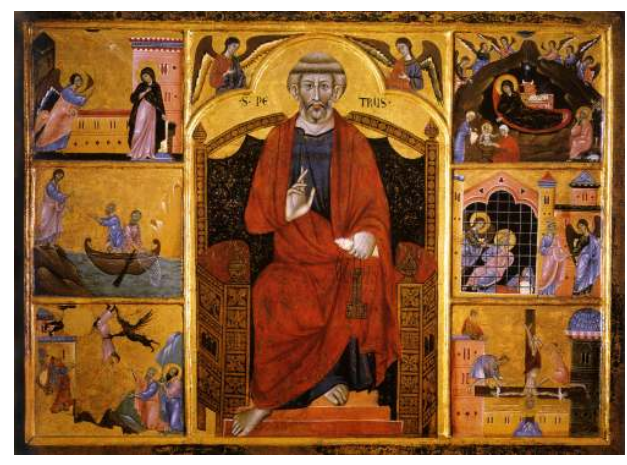
Angelico et Filippo Lippi. L'objectif est d'étudier l'évolution de chacune des figures constitutives de la scène - Marie, Jésus, Joseph, l'âne et le bœuf, les bergers, les sages-femmes, etc. - et surtout de la scène comme ensemble, à partir du motif figuratif du personnage agenouillé en adoration, toujours plus fréquent dans la Nativité au cours de la période et symptomatique des usages dévotionnels de l'image dans l'Italie médiévale.

2 Le titre, «La Nativité italienne : une histoire d'adoration (1250-1450) », rend hommage à L'Annonciation italienne. Une histoire de perspective (Paris, 1999) de Daniel Arasse ainsi qu'à sa démarche. Absent de l'iconographie de la Nativité jusqu'à la toute fin du Duecento, le geste de l'adoration - la position de prière qui consiste à se tenir à genoux les mains jointes, appelée genuflexio recta - y devient, en l'espace de quelques décennies à peine, un motif courant pratiqué aussi bien par Marie que par Joseph, les anges, les bergers, voire l'âne et le bœuf. L'hypothèse qui préside à ce travail est que ce geste dévotionnel, employé par les peintres comme outil figuratif, acquiert à l'égard de la Nativité une efficacité heuristique comparable à celle de la perspective pour l'Annonciation. Ainsi, si 
l'Annonciation est, du point de vue figuratif, la scène du mystère de l'Incarnation, la Nativité est la preuve de l'effective réalisation de ce dogme chrétien. Il incombe à l'Annonciation d'aider le fidèle à concevoir en esprit le sens de ce mystère, à la Nativité d'en montrer l'accomplissement et la reconnaissance. Le geste de l'adoration vient précisément signifier, dans le langage pictural, la reconnaissance: face au Dieu tout juste né, les êtres vivants s'agenouillent et, partant, se soumettent à sa Loi. Cette thèse se donne pour objet de retracer l'histoire de l'entrée puis de la propagation de l'adoration dans la Nativité. Ce geste devient à ce point central dans la scène qu'il finit même par la phagocyter: la Nativité devient ainsi Adoration des bergers, Adoration de l'Enfant. La question se pose alors de savoir quel rôle revêt(ent) le(s) personnage(s) orant(s) dans l'image autant que dans l'interaction entre l'image et le fidèle. L'irruption de ce motif dans le langage figuratif contribue en effet à la compréhension de l'usage dévotionnel des images.

3 Le geste de l'adoration est d'abord attribué par les peintres aux personnages de l'histoire sainte (Marie, Joseph) puis, dès le Trecento mais surtout au Quattrocento, à des personnages ajoutés à la scène, des saints ou bien des commanditaires. Cet ajout révèle que l'adoration fonctionne dans l'image comme le relais du geste de prière devant l'image. En plus de raconter l'histoire sainte, les Nativités tendent au fidèle un miroir - quel que soit le personnage en adoration dans l'image, Joseph, Marie, JeanBaptiste, les bergers, d'autres saints, des anges - dans lequel il peut se reconnaître en train de pratiquer l'adoration. L'objectif de ce travail est bien de montrer comment l'insertion du motif figuratif de l'adoration dans la Nativité constitue un support pour le transitus du dévot. Par un même terme, l'adoration désigne en somme une pratique (l'adoration de l'image), dont l'histoire et les doctrines sont retracées au chapitre 4, un motif iconographique - l'adoration dans l'image, c'est-à-dire la figure de l'orant -, qui est au centre de toute cette recherche, aussi bien qu'un type d'image dévotionnelle, enfin (l'Adoration).

Pour rendre compte des différentes dimensions de l'image - esthétique, sociale, historique, dévotionnelle, anthropologique -, l'iconographie sérielle, telle que la définit Jérôme Baschet dans L'iconographie médiévale (Paris, 2008), semble une méthode efficace. Par iconographie sérielle, on entend l'étude d'une même image en série, la série pouvant contenir plusieurs centaines d'éléments. Ce travail se fonde donc sur le recensement le plus exhaustif possible des Nativités, dont l'observation en série conduit à identifier des groupes homogènes, des tournants iconographiques, des tendances générales comme des cas exceptionnels. La thèse fait une place à chacun de ces cas de figure en exposant aussi bien des études quantitatives que des études de cas. Le site internet http://nativita.hypotheses.org/, construit pour permettre un accès permanent et public aux images qui constituent le fichier de la thèse, se voudrait un reflet de l'efficacité inhérente à la démarche sérielle.

5 Le premier chapitre recense et commente les textes par lesquels l'événement qu'est la Nativité (la naissance de Jésus) acquiert une dimension narrative tout en s'enrichissant, depuis les Évangiles canoniques jusqu'à la Légende dorée de Jacques de Voragine (XIII siècle), des personnages et des scènes secondaires que les peintres reprennent et remanient. Ceux-ci connaissent donc, sinon ces textes, en tout cas ces récits, aussi une partie du premier chapitre est-elle également consacrée à préciser le sens que l'on peut donner à cette compétence narrative dont les images font état. 
Par rapport au dernier texte analysé dans le premier chapitre (la Légende dorée), le deuxième fait un retour dans le temps, puisqu'il porte sur la mise en scène de la crèche par François d'Assise, le 24 décembre 1223 à Greccio, en Ombrie, en analysant d'abord la tradition textuelle qui retrace cet épisode, puis l'ensemble des images qui le commémorent. Les images de la Crèche à Greccio ne sont pas des Nativités auxquelles les peintres auraient ajouté la figure de saint François : s'ils y incluent quelques éléments de la Nativité - la crèche, l'âne et le bœuf uniquement -, ils les pensent avant tout comme des images dont l'iconographie, inédite, se rapporte aux usages dévotionnels et liturgiques de leur temps. Le rôle que François y tient est très variable, mais toujours problématique : chaque Crèche à Greccio est, en soi, une prise de position qui interroge la compatibilité de son invention par l'Assisiate avec la liturgie de Noël établie par l'Église romaine.

Fig. 1 - Antonio Vite, Saint François invente la crèche de Noël à Greccio en 1223, après 1386, fresque, Pistoia, San Francesco.

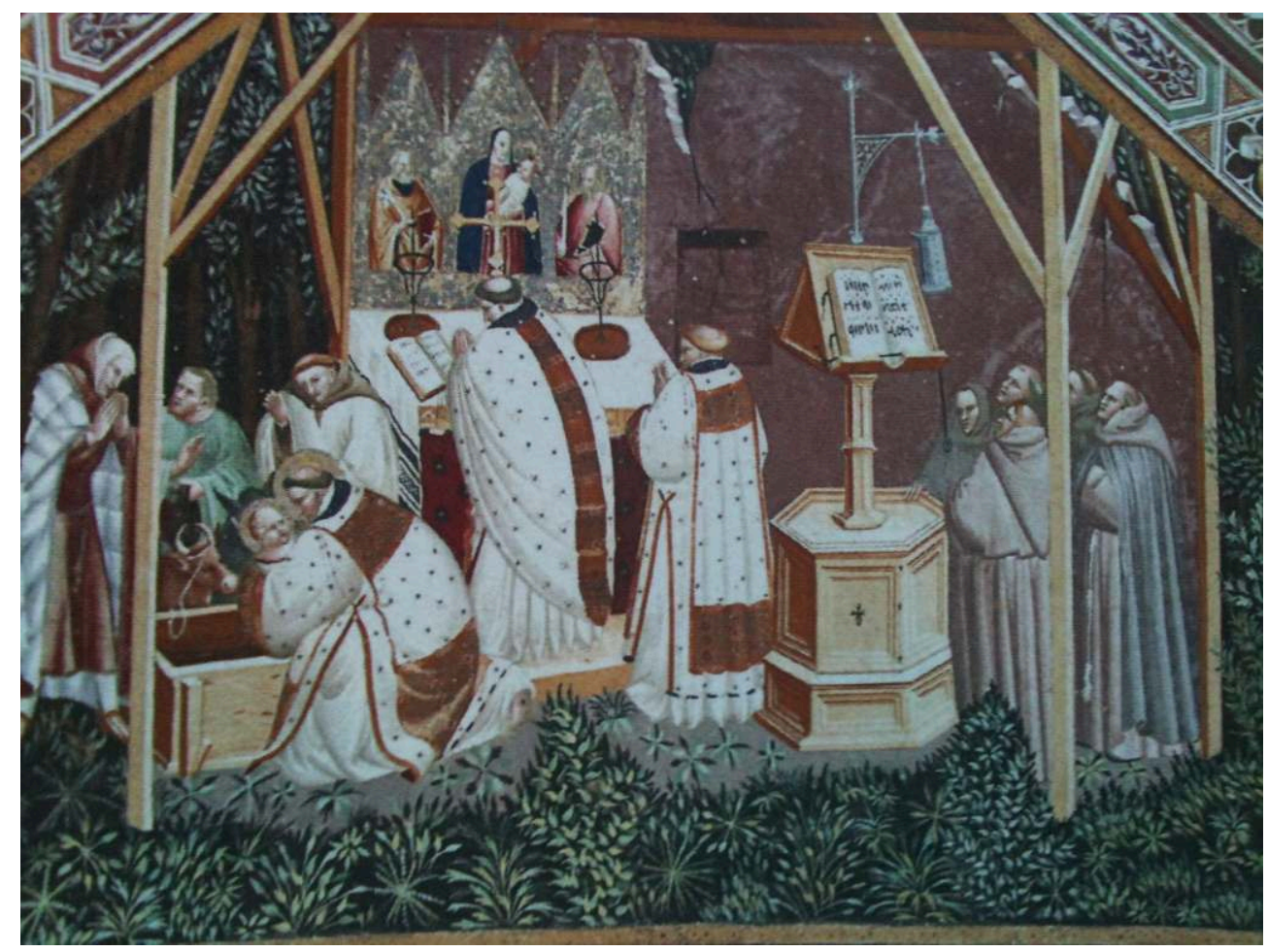

7 Le détour par l'ensemble, textuel et figuratif, de la Crèche à Greccio s'est en effet imposé à un double titre : parce qu'il illustre d'abord, à partir d'un exemple précis, l'intensité irréductiblement singulière du rapport entretenu par François avec la Nativité de l'Enfant; parce qu'il permet d'envisager l'impact que la dévotion franciscaine singularité exemplaire du fondateur ou imposition d'un modèle - va d'autre part exercer sur les composantes figuratives de la Nativité.

8 Avec le troisième chapitre commence donc l'exploration de la Nativité en tant que telle, précédée d'un aperçu sur l'histoire iconographique entre son apparition (au IV siècle) et le début de cette enquête (vers 1250). Depuis les motifs empruntés à l'art antique jusqu'à l'invention des scènes secondaires pouvant illustrer des dogmes chrétiens - les sages-femmes, par exemple, dont il est plus longuement question au chapitre 8 -, la Nativité passe, durant les siècles qui précèdent l'an Mil, du statut de figuration 
prophétique à celui de scène narrative. Dans l'art byzantin, la Nativité s'insère dans des cycles - de la Vie du Christ, de son Enfance, de la Vie de la Vierge -, et c'est de cette même manière que la conçoivent les peintres italiens du Duecento, dans les décors monumentaux à mosaïque ou à fresque comme dans les nombreuses scènes narratives qui entourent les figures en pied au centre des retables.

Fig. 2 - Guido di Graziano, Retable de saint Pierre (Nativité en haut à droite), 1280, or et détrempe sur bois, Sienne, Pinacothèque.

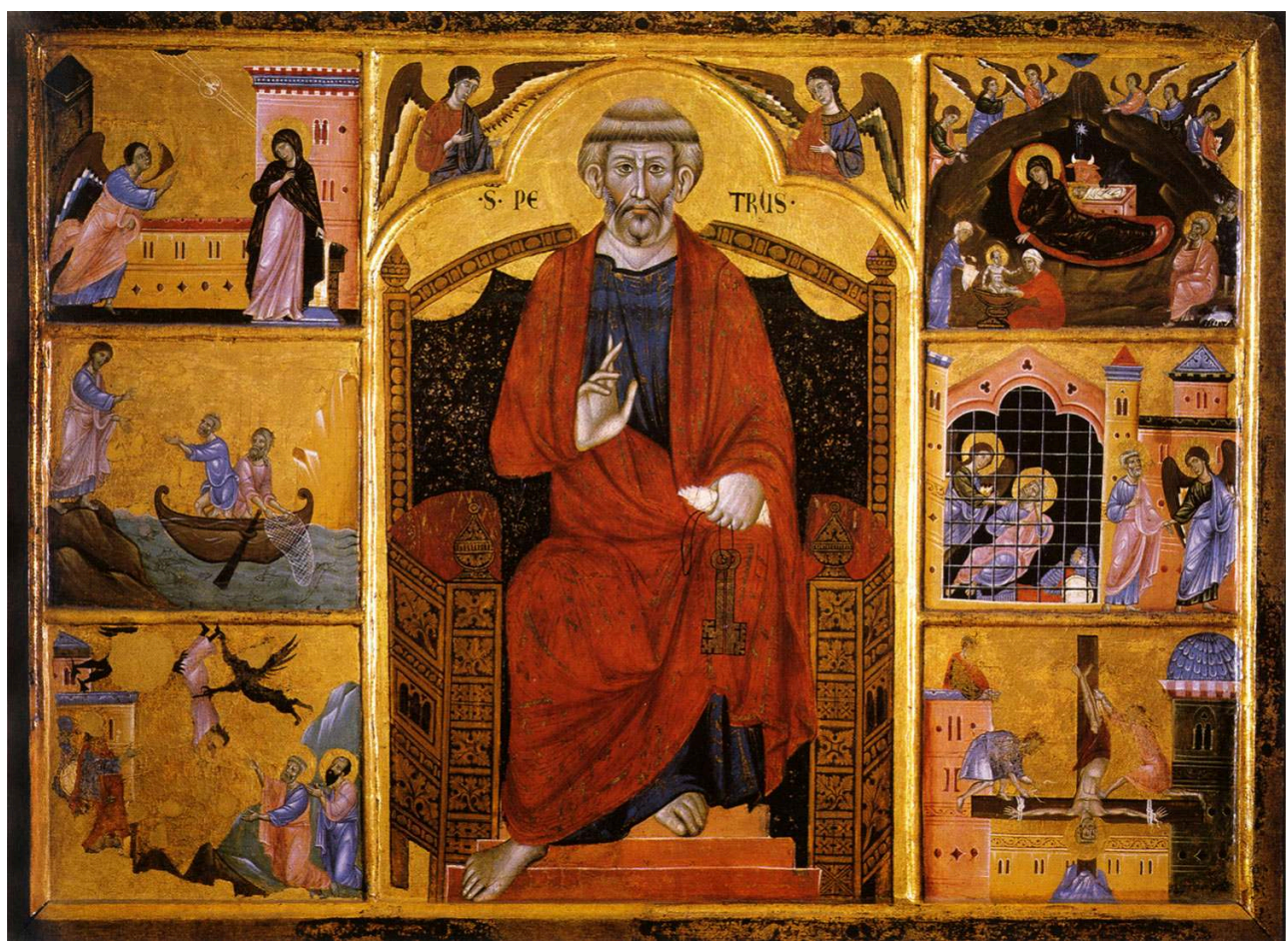

Dès le Duecento, les mosaïques commanditées par Nicolas IV pour les grandes basiliques de Rome, en vue du jubilé de 1300, font également apparaître la volonté de signifier iconographiquement son ancrage italien, et plus précisément romain. Dans ce processus d'appropriation de la Nativité, la dévotion franciscaine joue à son tour un grand rôle, qu'illustrent notamment les fresques de Giotto. De ce fait, les caractères figuratifs de la Nativité commencent à subir une altération de plus en plus évidente au tournant entre Duecento et Trecento, évoluant vers une représentation qui fait une large place au lien Mère Fils. Il ne s'agit en aucun cas de vider la scène de sa valeur théologique, mais l'expression de ce lien s'accompagne d'un changement iconographique dont l'importance est tout aussi grande : l'apparition de personnages en position d'adoration, justement chargés de visualiser la révérence due à l'Incarnation. Ce chapitre s'achève par l'analyse de plusieurs images hybrides de la Nativité et de l'Adoration des Mages, datant du premier tiers du Trecento, véritables témoignages visuels de l'entrée en scène du motif de l'adoration, sur lequel porte le chapitre suivant. 
Fig. 3 - Francesco da Rimini, Image hybride (Nativité, adoration des Mages, adoration des saints Joseph et François), env. 1330, détrempe sur bois, Coral Gables (Miami), Lowe Art Museum.

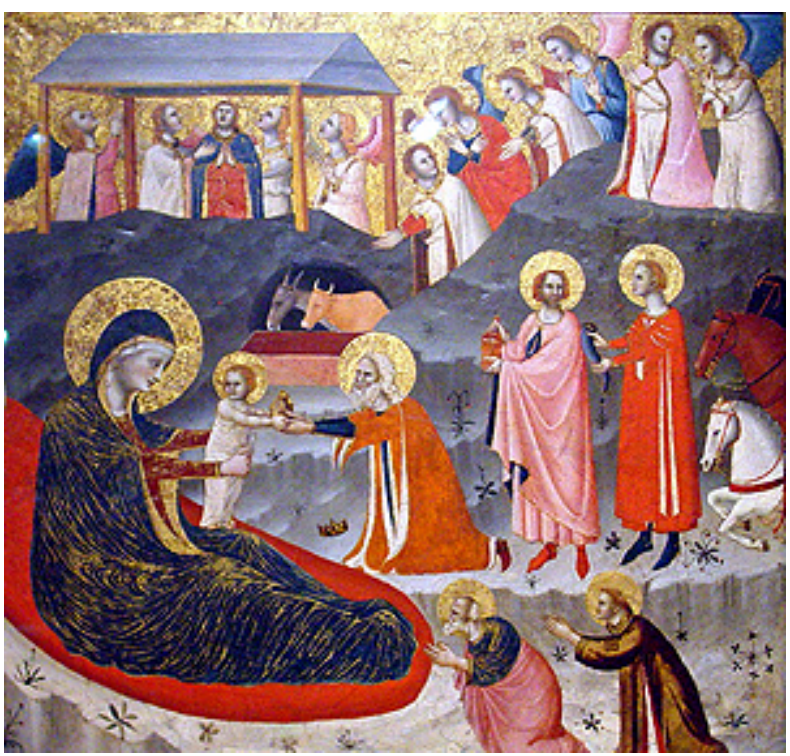

Le quatrième chapitre retrace d'abord la Querelle des images, la crise iconoclaste byzantine $\mathrm{du} \mathrm{VIII}^{\mathrm{e}}$ siècle et la production théologique concernant la licéité du culte des images jusqu'à Thomas d'Aquin. Parallèlement à cette histoire doctrinale de l'adoration, le propos se concentre sur l'élection, entre autres gestes dévotionnels, de la genuflexio recta comme position de prière archétypale au XIII ${ }^{\mathrm{e}}$ siècle. Un extrait des ricordanze d'un marchand florentin, Giovanni di Pagolo Morelli, permet de découvrir le détail d'une prière domestique se déroulant non seulement face à, mais aussi avec une image dévotionnelle. Le format le plus courant pour ces images de dévotion privée est celui des triptyques portatifs, qui constituent dans cette thèse un véritable corpus dans le corpus du fait de leur cohérence formelle et iconographique. 
Fig. 4 - Bernardo Daddi, Triptyque portatif (Nativité dans le volet gauche), 1338, argent, or et détrempe sur bois, Edimbourg, National Galleries of Scotland.

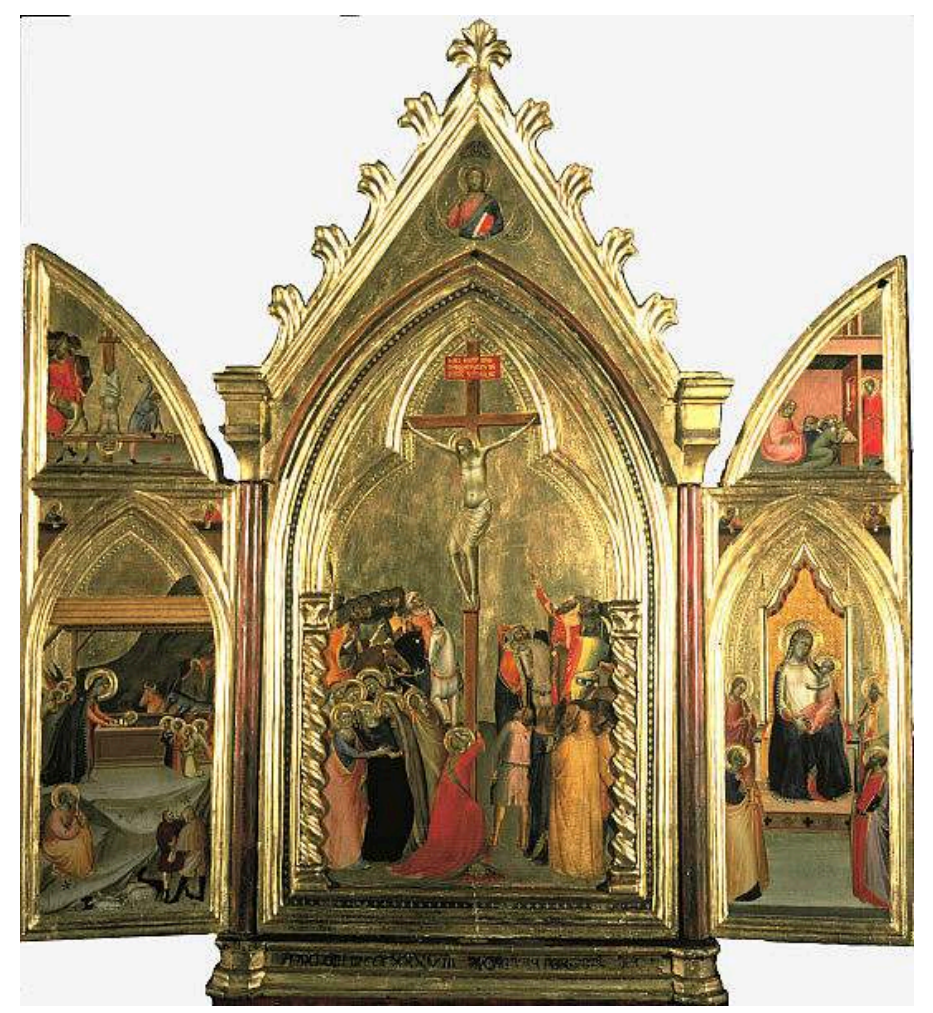

11 Ils se prêtent à une étude d'iconographie sérielle, menée dans la deuxième partie du chapitre 4, d'où il ressort qu'ils fonctionnent comme un laboratoire d'inventions figuratives. Au sein même d'un schéma remarquablement stable - la Nativité dans le volet gauche, la Vierge à l'Enfant dans le panneau central, la Crucifixion dans le volet droit -, les peintres expérimentent une gamme très large de variations dans la composition de la scène, où ce sont Marie et les bergers qui adoptent le plus rapidement la posture d'adoration. Le chapitre 4 s'achève par l'observation d'autres petits retables de dévotion privée dans lesquels, à un même moment historique, des Nativités à l'iconographie proche de celles du Duecento cohabitent avec des compositions à l'audace inventive spectaculaire, comme c'est le cas pour Ambrogio Lorenzetti.

Dans l'ensemble, le constat s'impose que les années 1300-1340 correspondent à un moment d'inventivité particulièrement fécond, dont participent pleinement les aquarelles du manuscrit italien 115 de la Bibliothèque nationale de France, analysées au chapitre 5. La Nativité y reçoit en effet un traitement figuratif exceptionnellement développé. Tout un ensemble d'images alterne sur les folia du manuscrit avec le texte des Meditationes Vitae Christi, écrit par le franciscain Jean de Caulibus (1335-1340) à l'intention d'une clarisse qu'il devait former. Cette dernière est invitée à méditer sur le texte autant que devant les images, voire à y entrer - et les figures peintes, elles-mêmes disposées face au spectateur, tendent la main en signe d'accueil. Le texte, et les illustrations davantage encore, dilate la Nativité dans toute son extension temporelle, depuis une aquarelle de Marie au ventre rond, sur le point d'accoucher, jusqu'à plusieurs scènes de l'après accouchement. 
Fig. 5 - Anonyme toscan, Nativité, 1335-1340, aquarelle, PARIS, BnF, ms. it. 115, fol. 19.

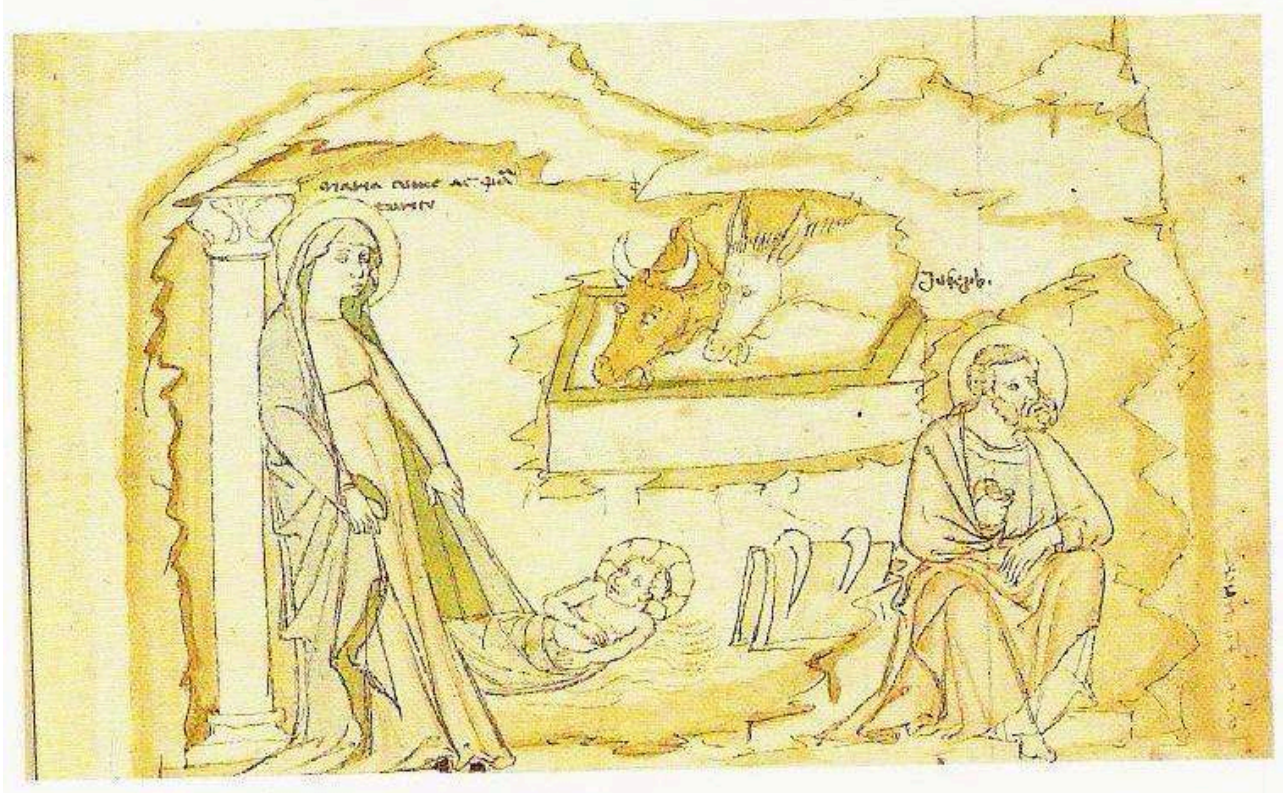

L'un des intérêts du ms. it. 115 est donc d'observer les interactions variables entre texte et peinture, mais il est loin d'être le seul. Jean de Caulibus y consigne en effet la version franciscaine du récit de la Nativité qu'il aurait reçue d'un autre franciscain, à qui elle aurait été révélée par la Vierge en personne. Cette présentation sous forme de témoignage rapporté est destinée à légitimer son récit, qu'il ordonne autour des différentes adorations pratiquées par tous les êtres (animaux, humains, anges). Son texte est ainsi le premier qui subordonne le récit de la Nativité à la scansion répétée de ce geste dévotionnel.

Quelques décennies plus tard, Brigitte de Suède (1303-1373) produit un nouveau récit de la Nativité, objet du chapitre $6:$ il découle à son tour d'une révélation de la Vierge qui est survenue, pour elle, dans la grotte même de Bethléem, au cours de son pèlerinage de 1372. Il s'agit, cette fois-ci, du premier récit où accouchement et position d'adoration se confondent. Dans le cadre d'abord de son procès de canonisation, puis à l'initiative des milieux dévots à la sainte, canonisée en 1391, plusieurs retables illustrent cette révélation, qui confèrent tous un double statut figuratif à la scène. Si l'on voit, en effet, au centre de l'image, une Nativité dont des détails récurrents renvoient au texte brigittin, la figure de Brigitte, agenouillée sur un côté de la composition, à l'extérieur de la grotte, dit bien que c'est sa vision qui est alors représentée. 
Fig. 6 - Niccolò di Tommaso, Sainte Brigitte de Suède a une vision de la Nativité, 1373-1375, détrempe sur bois, Vatican, Pinacothèque.

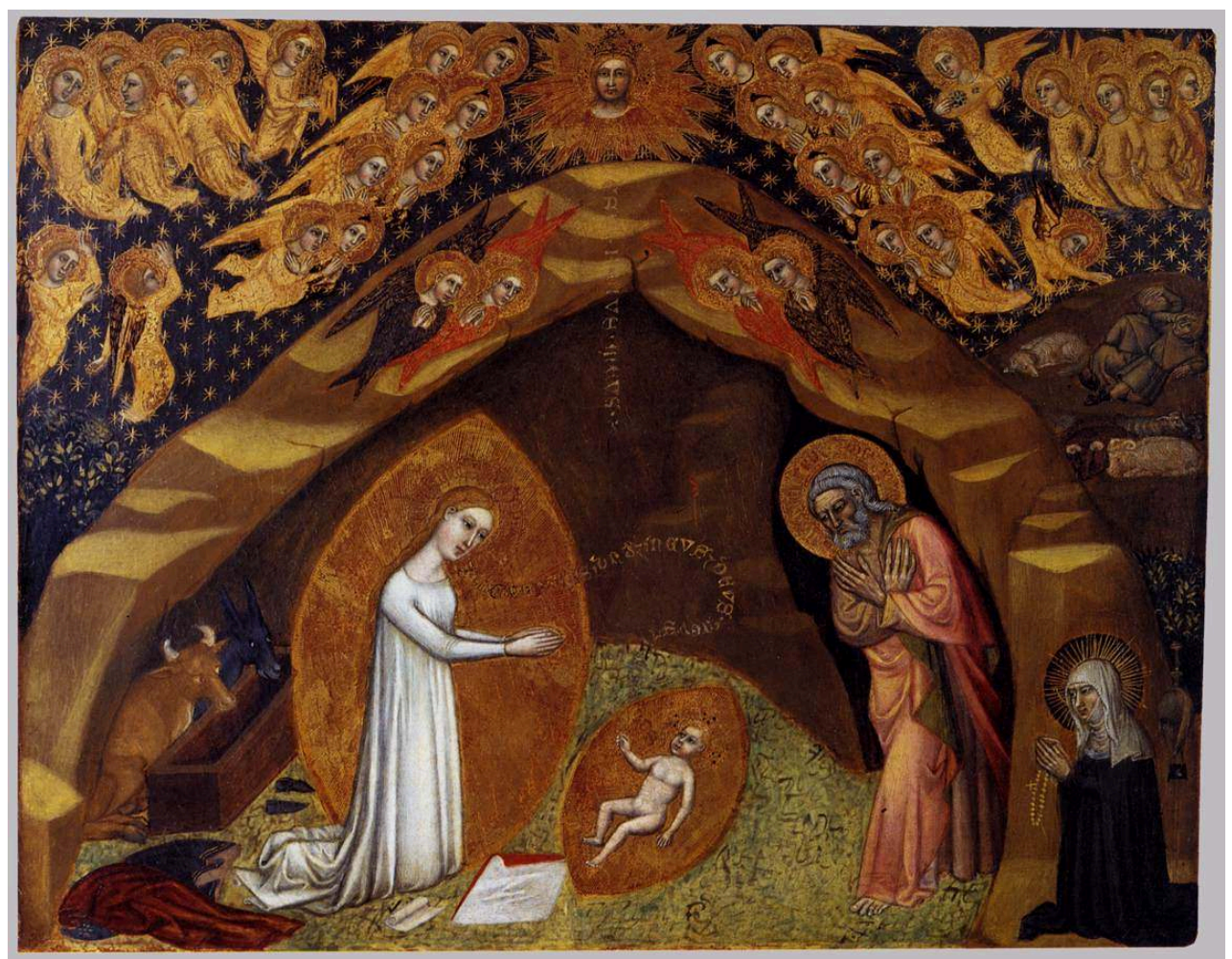

Ainsi, dans les Nativités avec Brigitte de Suède, une figure adorante de fidèle illustre, en outre, pour la première fois, dans la composition même, un nouveau rapport personnalisé à l'image peinte. Dans l'ensemble des Nativités, il semble bien que ce soient les figures des bergers, désormais de plus en plus souvent agenouillés au premier plan, qui permettent de généraliser cette aspiration à la personnalisation de la dévotion par le truchement de l'image, ce que le chapitre 7 s'attache à illustrer.

16 Jusqu'au début du Trecento, les bergers font l'objet d'une scène secondaire de la Nativité, l'Annonce, lors de laquelle un ange les informe de la naissance du Sauveur et leur enjoint de se rendre auprès de lui. Le résultat de cette injonction - les bergers devant la crèche, rendant hommage au Nouveau né - est en fait une invention picturale de Taddeo Gaddi, aux alentours de 1330. Les bergers, ainsi intégrés au moment principal de l'action, accèdent au premier plan de la scène, qui se trouve altérée, dans sa nature même, par ce changement. La Nativité se transforme en Adoration des bergers et le véritable sujet de la scène devient la dévotion portée par les bergers, archétypes tantôt du pauvre, tantôt du pèlerin, dans tous les cas de personnages facilitant l'identification des fidèles. 
Fig. 7 - Bartolo di Fredi, Adoration des bergers, 1374, détrempe sur bois, New York, Metropolitan Museum of Art.

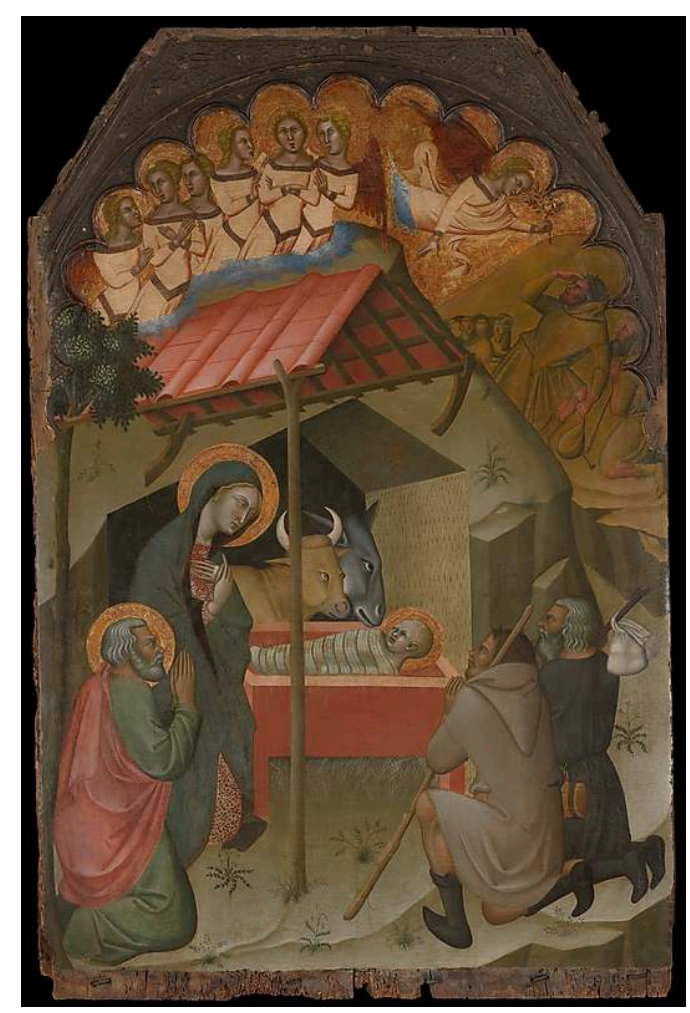

L'agenouillement des bergers se manifeste rapidement comme un acte de dévotion envers Marie et ce n'est sans doute pas un hasard si les peintres siennois sont parmi les penseurs les plus assidus de cette scène nouvelle.

L'occupation du premier plan par les bergers ainsi que le soin apporté à représenter la dévotion mariale ont, entre autres conséquences, favorisé la raréfaction des sagesfemmes et, partant du Bain de l'Enfant, dans la Nativité où leur présence était porteuse, depuis l'origine, d'un sens théologique dont l'histoire est retracée dans le chapitre 8. Les évangiles apocryphes rapportent, en effet, que deux sages-femmes se rendent au chevet de la jeune accouchée : l'une croit immédiatement en la naissance miraculeuse, l'autre voit son bras brûler pour avoir voulu s'assurer de la virginité de Marie. Renseignée par un ange, elle touche ensuite l'Enfant, se convertit et récupère son bras. De cet épisode, présent dans l'iconographie de la Nativité des premiers siècles, il ne reste presque plus trace au Duecento. Les sages-femmes sont alors représentées en train de donner à l'Enfant un bain qui est en fait une préfiguration du baptême dans le Jourdain. La tendance générale, sauf exceptions notables chez Taddeo Gaddi et Gentile da Fabriano, est à l'exclusion progressive de ces figures de la Nativité de Jésus et à leur report dans les Nativités de Marie. Ainsi, la peinture du Tre et du Quattrocento fonctionnant volontiers par cycles, les deux Nativités deviennent aussi distinctes que complémentaires : celle de Jésus relève de l'exception, celle de Marie, au contraire, s'insère dans une scène de genre qui, comme en témoignent, notamment, les scènes peintes sur des deschi da parto, reproduit les circonstances d'une naissance dans l'univers domestique. De son côté, la Nativité de Jésus évolue dans une direction qui s'éloigne de ces usages anthropologiques de l'image de la naissance : d'autant plus que, au début du Quattrocento, s'élabore l'invention d'une scène dont la valeur symbolique, 
qui entraîne la redistribution des membres de la sainte Famille dans la composition, aboutit à l'exhibition du corps nu de l'Enfant.

Fig. 8 et 9 - Giovanni Francesco da Rimini, Nativité de Marie et Nativité de Jésus, env. 1445, Paris, Louvre.
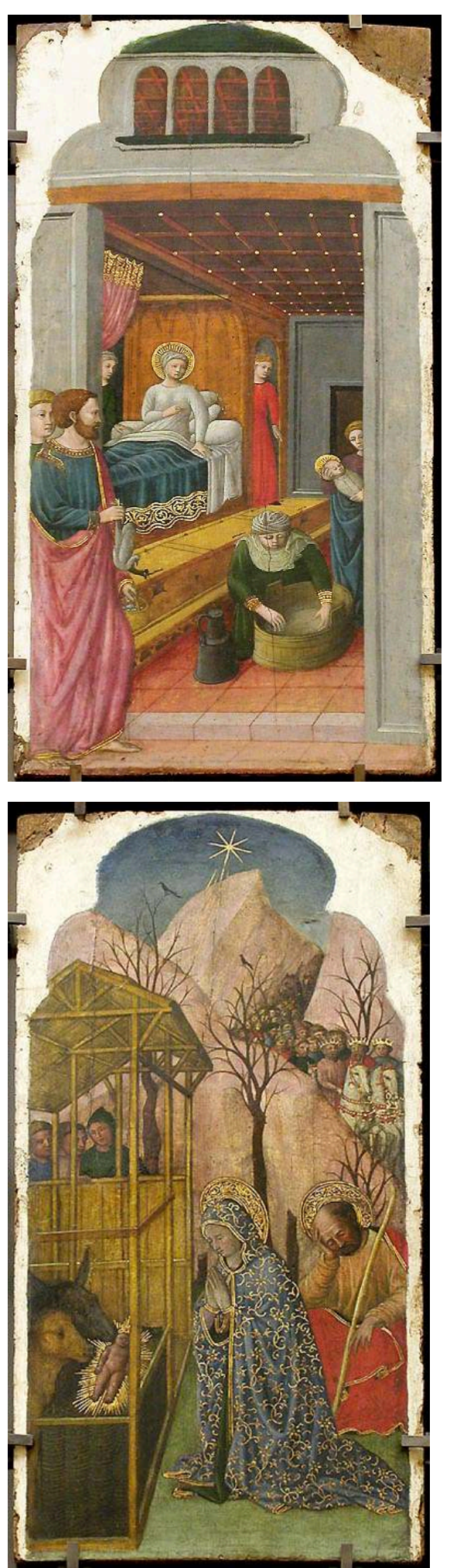

Bulletin du centre d'études médiévales d'Auxerre | BUCEMA, 17.2 | 2013 
Après avoir suivi, dans les chapitres 7 et 8 , les parcours divergents des personnages secondaires (bergers et sages-femmes) qui accèdent au premier plan (les bergers) ou sont expulsés du cadre (les sages-femmes), le dernier chapitre s'attache à analyser les modifications qui affectent les éléments constitutifs d'une Nativité : à commencer par le lieu, la grotte, où l'événement s'est produit. Les nombreuses Nativités que Lorenzo Monaco peint dans des prédelles de polyptyques durant les premières décennies du Quattrocento traduisent une méditation sans précédent sur la valeur théologique de la scène et la capacité du langage figuratif à l'illustrer. Il est ainsi l'un des premiers à tenter d'intégrer un bâtiment en ruine à la Nativité, motif qui devient ensuite caractéristique de la scène au Quattrocento, car il permet de montrer comment la Nouvelle Loi (l'Église de Jésus) terrasse les ruines de l'Ancienne - les temples juifs et païens. Mais Lorenzo Monaco offre également une représentation tout à fait unique de Joseph qui exige, pour être expliquée, de retracer l'histoire de son déploiement dans la Nativité, de la silhouette recroquevillée et songeuse à la figure du père terrestre participant à la vie du Christ ici-bas.

Fig. 10 - Lorenzo Monaco, Nativité, 1409, or et détrempe sur bois, New York, Metropolitan Museum of Art.

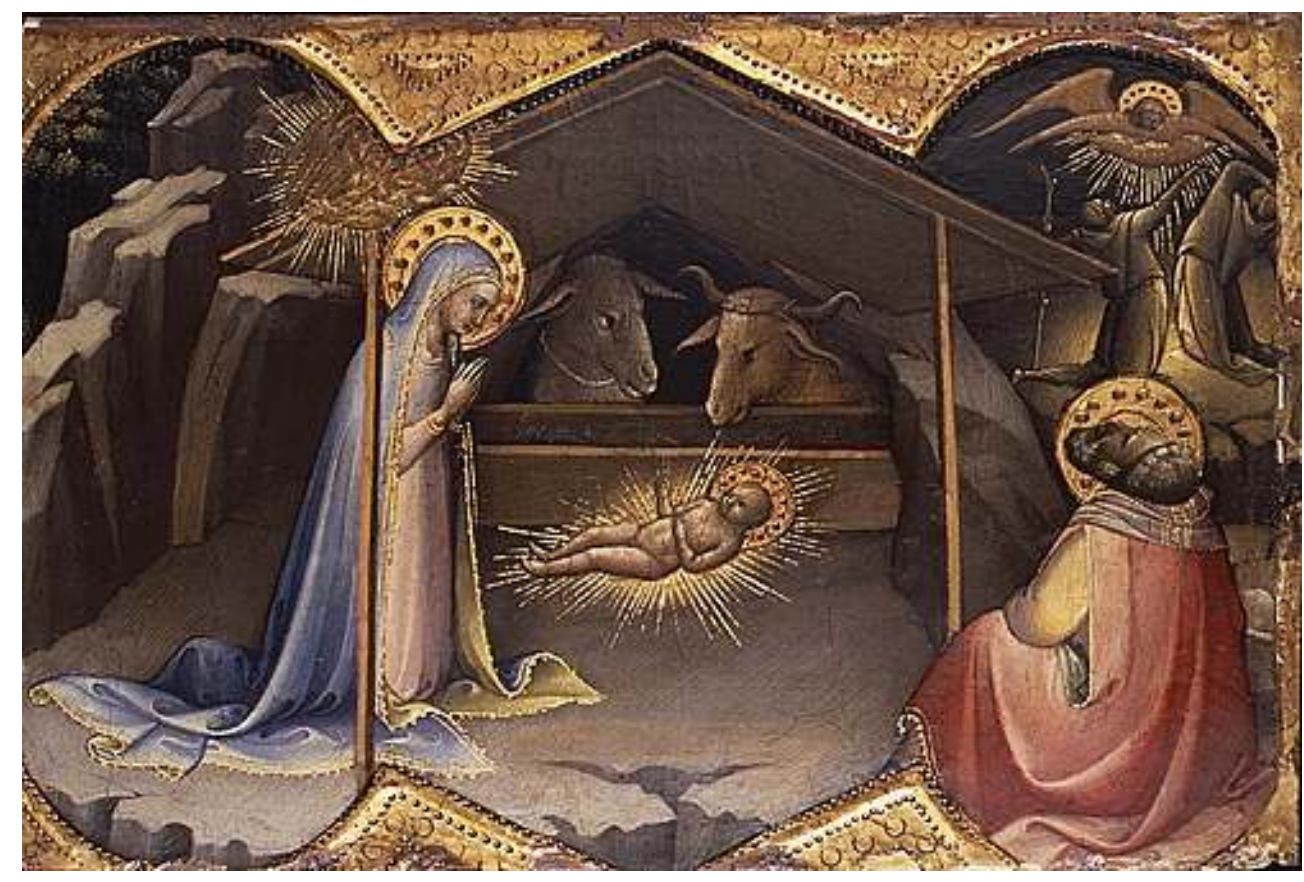

Ce dernier est progressivement extrait de la crèche par les peintres qui l'exposent nu au premier plan, offert à la dévotion de tous. Ce changement implique que l'on redéfinisse, à son tour, la fonction de l'âne et du bœuf dans l'image, désormais éloignés de l'Enfant qu'ils réchauffaient auparavant; mais il comporte aussi une nouvelle disposition de Marie et de Jésus dans l'espace. La Mère et le Fils sont en effet pris dans un rapport dialectique, où la première doit à la fois offrir au regard dévot et au sacrifice expiatoire le second, en même temps qu'elle le protège. Ce rapport s'exprime figurativement par l'usage des textiles avec lesquels Marie voile et dévoile Jésus. L'Adoration de l'Enfant par sa Mère semble répondre de mieux en mieux aux demandes d'images dévotionnelles aux alentours de 1450, et les traits de la Nativité - Joseph, les animaux, la crèche - tendent à y occuper progressivement une place moindre, relégués à l'arrière-plan, voire éliminés. La place prépondérante que prend l'Adoration de l'Enfant 
par sa Mère altère progressivement la nature même de la scène, pliant la narration aux besoins de la dévotion.

Fig. 11 - Giovanni di Paolo, Adoration de l'Enfant, 1452-1458, détrempe sur bois, Cambridge (MA), Harvard Art Museums/Fogg Museum.

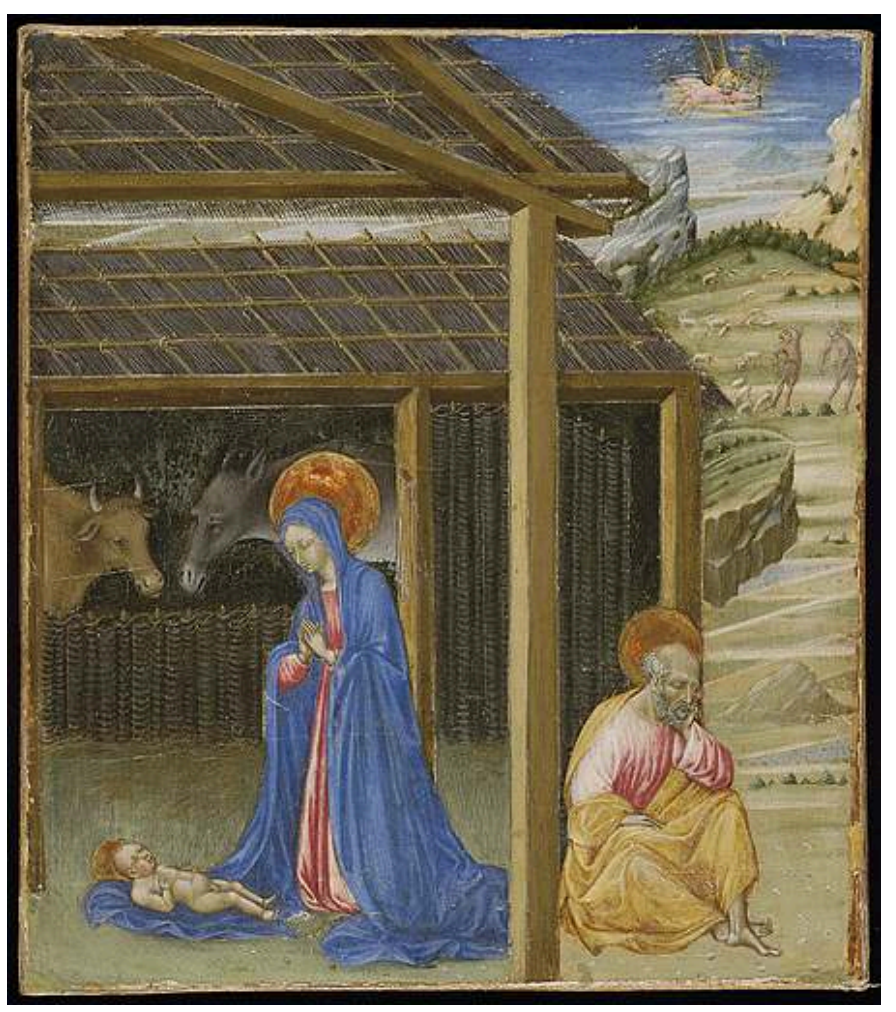

21 Le couple Mère Fils devient, en réalité, un objet de représentation et de dévotion autonome : l'Adoration de l'Enfant, à partir des années 1450, provient de la Nativité, mais n'en est plus une.

En définitive, l'étude sérielle permet de constater avec quelle fréquence des Nativités étaient peintes entre 1250 et 1450, sans la moindre interruption, constituant une véritable tradition iconographique. Dans le même temps apparaît la remarquable mobilité des différents motifs iconographiques qui composent la scène et dont aucun ne demeure stable. L'observation d'une même scène sur la longue durée contraint à relativiser l'idée d'une identité intrinsèque de l'image ou, pour le dire autrement, de reconductibilité à un "modèle " de la Nativité. Ce qui ressort le plus fortement, au contraire, est la pertinence qu'il y a, par le truchement d'une observation détaillée des images, à percer la pensée du peintre à l'œuvre dans la composition et à reconstituer la motivation qui conduit tel ou tel peintre à altérer de telle ou de telle autre manière un ou plusieurs éléments de la Nativité en fonction de la compréhension théologique et dévotionnelle qu'il en a. Le peintre est, en effet, détenteur de la capacité - qu'il exploite plus ou moins à plein selon les moyens à sa disposition - d'entraîner l'œil, puis le cœur et l'esprit du dévot dans une méditation dont ce dernier attend un soulagement et une efficacité.

Reçu : 17 septembre 2013 - Accepté : 31 octobre 2013 
INDEX

Mots-clés : Nativité, crèche, adoration, bergers, sage-femme, iconographie sérielle, retable, polyptyque, pensée figurative, pratique dévotionnelle, Giotto, Taddeo Gaddi, Giovanni di Pagolo Morelli, Jean de Caulibus, Brigitte de Suède, Niccolò di Tommaso, Lorenzo Monaco, François d'Assise (saint)

\section{AUTEUR}

\section{GIULIA PUMA}

Centre d'étude et de recherche sur la littérature italienne médiévale [CERLIM]. Sorbonne Nouvelle - Paris 3 